\title{
Electronic Northern Analysis of Genes and Modeling of Gene Networks Underlying Bovine Milk Fat Production
}

\author{
Bhaskar Ganguly, Tanuj Kumar Ambwani, and Sunil Kumar Rastogi \\ Animal Biotechnology Center, Department of Veterinary Physiology and Biochemistry, College of Veterinary and Animal Sciences, \\ G. B. Pant University of Agriculture and Technology, Pantnagar 263145, India
}

Correspondence should be addressed to Bhaskar Ganguly; vetbhaskar@gmail.com

Received 22 August 2017; Accepted 17 October 2017; Published 7 November 2017

Academic Editor: Norman A. Doggett

Copyright (C) 2017 Bhaskar Ganguly et al. This is an open access article distributed under the Creative Commons Attribution License, which permits unrestricted use, distribution, and reproduction in any medium, provided the original work is properly cited.

\begin{abstract}
Milk fat is one of the most important economic traits in dairy animals. Yet, the biological machinery involved in milk fat synthesis remains poorly understood. In the present study, expression profiling of 45 genes involved in lipid biosynthesis and secretion was performed using a computational approach to identify those genes that are differentially expressed in mammary tissue. Transcript abundance was observed for genes associated with nine bioprocesses, namely, fatty acid import into cells, xenobiotic and cholesterol transport, acetate and fatty acid activation and intracellular transport, fatty acid synthesis and desaturation, triacylglycerol synthesis, sphingolipid synthesis, lipid droplet formation, ketone body utilization, and regulation of transcription in mammary, skin, and muscle tissue. Relative expression coefficient of the genes was derived based on the transcript abundance across the three tissue types to determine the genes that were preferentially expressed during lactation. 13 genes (ACSS1, ACSS2, ADFP, CD36, FABP3, FASN, GPAM, INSIG1, LPL, SCD5, SPTLC1, SREBF1, and XDH) showed higher expression in the mammary tissue of which 6 (ADFP, FASN, GPAM, LPL, SREBF1, and XDH) showed higher expression during adulthood. Further, interaction networks were mapped for these genes to determine the nature of interactions and to identify the major genes in the milk fat biosynthesis and secretion pathways.
\end{abstract}

\section{Introduction}

Milk fat content is regarded as one of the most important economic traits of milch animals; identification of gene networks that regulate lipid biosynthesis and secretion in the mammary gland is essential to our understanding of lactation physiology. Finding candidate genes for improved fat content represents a constant research goal [1] that may further provide opportunities for genetic manipulations to derive more or better milk fat. Comparing biomolecular composition of mammary tissue with other tissues can allow insights into the molecular responses that govern milk fat production. Transcriptional regulation is a major long-term mechanism for the control of metabolism, and switching on and off gene expression essentially drives a cell's biological function and activity [2]. In the present study, an attempt has been made to identify the genes, which are differentially expressed during milk fat production in bovines, and determine their interaction networks using a computational approach.

\section{Materials and Methods}

2.1. Identification of Differentially Expressed Genes. The reference bovine gene sequences for 45 genes, previously known to be involved in lipid synthesis (Table 1) [3], were obtained from Ensembl [4]. Electronic Northern (e-Northern) was performed using dbEST and UniGene; briefly, the dbEST [5] was queried for these sequences by BLASTN v2.2.27 [6] using default parameters and the significant hits were looked up in UniGene ESTProfile [7] for transcript abundance based on normalized "transcripts per million" (TPM) values in mammary tissue $\left(\mathrm{TPM}_{\mathrm{ma}}\right)$, skin $\left(\mathrm{TPM}_{\mathrm{s}}\right)$, and muscles $\left(\mathrm{TPM}_{\mathrm{mu}}\right)$. Where information was available, transcript 
TABLE 1: Genes involved in milk fat synthesis and secretion. 45 genes previously reported to be involved in nine different bioprocesses (in bold) of milk fat biosynthesis and secretion [3] were studied.

\begin{tabular}{|c|c|}
\hline Gene name & Gene product \\
\hline \multicolumn{2}{|c|}{ (1) FA import into cells } \\
\hline CD36 & CD36 molecule (thrombospondin receptor) \\
\hline$L P L$ & Lipoprotein lipase \\
\hline$V L D L R$ & Very-Low-Density Lipoprotein Receptor \\
\hline \multicolumn{2}{|c|}{ (2) Xenobiotic and Cholesterol transport } \\
\hline$A B C A 1$ & ATP-binding cassette, subfamily A (ABC1), member 1 \\
\hline ABCG2 & ATP-binding cassette, subfamily G (WHITE), member 2 \\
\hline \multicolumn{2}{|c|}{ (3) Acetate and FA activation and intracellular transport } \\
\hline$A C B P$ & Acyl-CoA binding protein (diazepam binding inhibitor) \\
\hline ACSL1 & Acyl-CoA synthetase long-chain family member 1 \\
\hline ACSS1 & Acyl-CoA synthetase short-chain family member 1 \\
\hline ACSS2 & Acyl-CoA synthetase short-chain family member 2 \\
\hline FABP3 & Fatty acid-binding protein, heart \\
\hline \multicolumn{2}{|c|}{ (4) Fatty acid synthesis and desaturation } \\
\hline ACACA & Acetyl-coenzyme A carboxylase alpha \\
\hline FADS1 & Fatty acid desaturase 1 (delta- 5 desaturase) \\
\hline FADS2 & Fatty acid desaturase 2 (delta- 6 desaturase) \\
\hline FASN & Fatty acid synthase \\
\hline SCD5 & Stearoyl-CoA desaturase (delta-9-desaturase) \\
\hline \multicolumn{2}{|c|}{ (5) Triacylglycerol synthesis } \\
\hline AGPAT6 & 1-Acylglycerol-3-phosphate O-acyltransferase 6 \\
\hline DGAT1 & Diacylglycerol acyltransferase 1 \\
\hline DGAT2 & Diacylglycerol acyltransferase 2 \\
\hline GPAM & Glycerol-3-phosphate acyltransferase, mitochondrial \\
\hline LPIN1 & Lipin 1 \\
\hline \multicolumn{2}{|c|}{ (6) Sphingolipid synthesis } \\
\hline$A S A H L$ & $\mathrm{~N}$-Acylsphingosine amidohydrolase-like \\
\hline LASS2 & LAG1 homolog, ceramide synthase 2 \\
\hline$O S B P$ & Oxysterol-binding protein 1 \\
\hline OSBPL10 & Oxysterol-binding protein-like 10 \\
\hline OSBPL 2 & Oxysterol-binding protein-like 2 \\
\hline SGPL1 & Sphingosine-1-phosphate lyase \\
\hline SPHK2 & Sphingosine kinase 2 \\
\hline SPTLC1 & Serine palmitoyltransferase, long-chain base subunit 1 \\
\hline SPTLC2 & Serine palmitoyltransferase, long-chain base subunit 2 \\
\hline$U G C G$ & Ceramide glucosyltransferase \\
\hline \multicolumn{2}{|c|}{ (7) Lipid droplet formation } \\
\hline$A D F P$ & Adipose differentiation related protein (adipophilin, PLIN2) \\
\hline BTN1A1 & Butyrophilin, subfamily 1 , member A1 \\
\hline PLIN & Perilipin \\
\hline$X D H$ & Xanthine dehydrogenase \\
\hline \multicolumn{2}{|c|}{ (8) Ketone body Utilization } \\
\hline BDH1 & 3-Hydroxybutyrate dehydrogenase, type 1 \\
\hline OXCT1 & 3-Oxoacid CoA transferase 1 \\
\hline \multicolumn{2}{|c|}{ (9) Regulation of transcription } \\
\hline INSIG1 & Insulin-induced gene 1 \\
\hline INSIG2 & Insulin-induced gene 2 \\
\hline$P P A R G$ & Peroxisome proliferator-activated receptor gamma \\
\hline PPARGCIA & PPAR gamma, coactivator 1 alpha \\
\hline PPARGC1B & PPAR gamma, coactivator 1 beta \\
\hline SCAP & SREBP cleavage activating protein \\
\hline
\end{tabular}


TABLE 1: Continued.

\begin{tabular}{cl}
\hline Gene name & Gene product \\
\hline SREBF1 & Sterol regulatory element-binding transcription factor 1 \\
SREBF2 & Sterol regulatory element-binding transcription factor 2 \\
THRSP & Thyroid hormone responsive SPOT14 \\
\hline
\end{tabular}

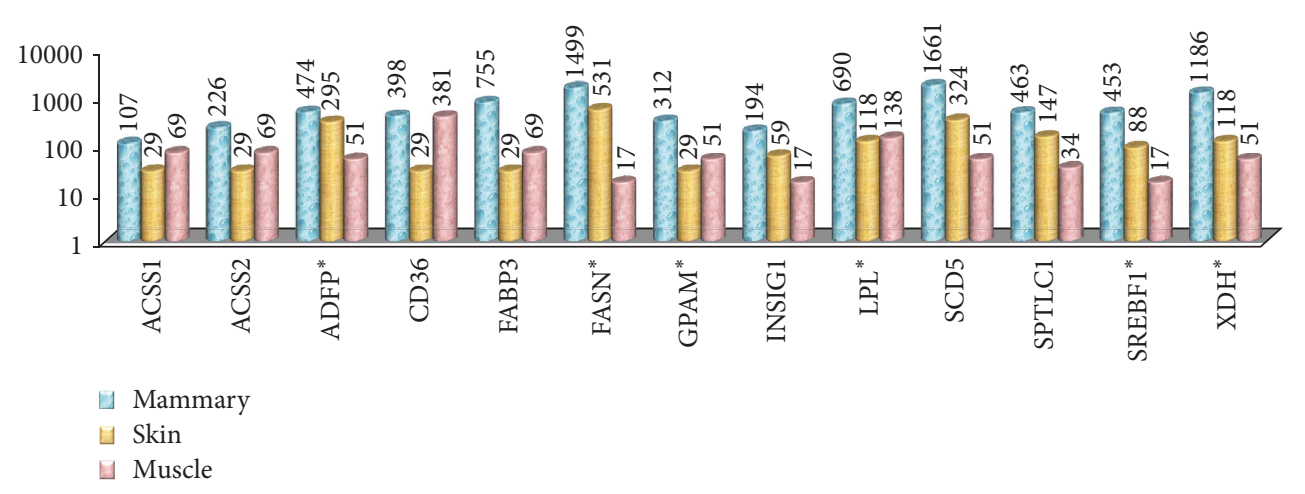

FIGURE 1: Transcript abundance of overexpressed genes. Based on $\mathrm{TPM}_{\mathrm{ma}}: \mathrm{TPM}_{\mathrm{s}}$ and $E_{r}$ values, 13 genes appeared to be overexpressed in mammary tissue. Transcript abundance values for these genes in mammary tissue, skin, and muscle have been shown for comparison. Genes marked with “*” showed preferential expression in adult-derived tissues.

abundance (value not shown) was also compared between adult and young stages.

Percent mammary transcript abundance for a gene $x$ was calculated using the formula:

$\%$ Transcript abundance

$$
=\left[\frac{\mathrm{TPM}_{\mathrm{ma}} x}{\sum \mathrm{TPM}_{\mathrm{ma}} \text { all genes }}\right] \times 100 \text {. }
$$

To confirm preferential mammary expression, relative expression coefficient $\left(E_{r}\right)$ was calculated as the ratio of mammary transcript abundance to the geometric mean of cutaneous and muscle transcript abundance; that is,

$$
E_{r}=\frac{\mathrm{TPM}_{\mathrm{ma}}}{\sqrt{ }\left(\mathrm{TPM}_{\mathrm{s}} \cdot \mathrm{TPM}_{\mathrm{mu}}\right)} .
$$

A twofold change in $E_{r}$ was, arbitrarily, assumed to be significant; that is, upregulation of expression was inferred when $\mathrm{TPM}_{\mathrm{ma}}>\mathrm{TPM}_{\mathrm{s}}$ and $E_{r} \geq 2$. Similarly, downregulation was inferred when $\mathrm{TPM}_{\mathrm{ma}}<\mathrm{TPM}_{\mathrm{s}}$ and $E_{r} \leq 0.5$.

2.2. Gene Network Analysis. Interaction networks and coexpression profiles for the genes were derived using STRING $v 9.1$ with default settings [8]. STRING is a web-based application for network generation and visualization that uses a database of physical and functional protein interactions derived from four separate sources, namely, genomic context, high-throughput experimental data, coexpression, and existing literature. It quantitatively combines the information from these four sources to generate a weighted interaction network.

\section{Results and Discussion}

3.1. Transcript Abundance. Transcript abundance was inferred from UniGene ESTProfile on the basis of normalized TPM values (Table 2) for the 45 genes involved in nine bioprocesses including fatty acid import into cells (CD36, $L P L$, and $V L D L R$ ); xenobiotic and cholesterol transport (ABCA1, $A B C G 2$ ); acetate and fatty acid activation and intracellular transport (ACSS1, ACSS2, ACSL1, ACBP, and FABP3); fatty acid synthesis and desaturation (ACACA, FADS1, FADS2, $F A S N$, and SCD5); triacylglycerol synthesis (AGPAT6, DGAT1, DGAT2, GPAM, and LPIN1); sphingolipid synthesis (ASAHL, LASS2, OSBP, OSBPL10, OSBPL2, SGPL1, SPHK2, $S P T L C 1, S P T L C 2$, and UGCG); lipid droplet formation (ADFP, BTN1A1, PLIN, and XDH); ketone body utilization (BDH1, OXCT1); and transcriptional regulation (INSIG1, INSIG2, PPARG, PPARGCIA, PPARGCIB, SCAP, SREBF1, $S R E B F 2$, and THRSP). Of the 45 genes included in the study, 23 genes did not have complete ESTProfiles and hence could not be included for further analysis. Notably, the absence of ESTProfiles of these 23 genes does not depress the robustness of the methodology that has been employed in the present study. Clearly, as more and more ESTProfiles get submitted to UniGene, it would become possible to use the same approach for analyzing the expression patterns of different genes including those of these 23 genes. Further, though ESTProfile TPM values lack exactitude as a measure of gene expression, the differences in TPM values tend to correlate with overall expression patterns.

3.2. $T P M_{m a} / T P M_{s}$ and Percent Transcript Abundance: Functional Inferences. Based on $E_{r}$ and $\mathrm{TPM}_{\mathrm{ma}}: \mathrm{TPM}_{\mathrm{s}}$ values, 13 genes (ACSS1, ACSS2, ADFP, CD36, FABP3, FASN, GPAM, INSIG1, LPL, SCD5, SPTLC1, SREBF1, and XDH; Table 2; Figure 1) were found to exhibit higher mammary expression 
TABLE 2: Summary of results of transcript abundance studies. Of the 45 genes involved, 23 genes (S. numbers " $23-45$ ”) did not have complete UniGene ESTProfile and were precluded from further studies. Of the 22 genes studied (S. numbers "1-22"), 13 genes (in bold) appeared to be overexpressed in mammary tissue. Of these, six genes (marked with an asterisk) further showed preferential expression in adult-derived tissues. TPM: transcripts per million; ma: mammary; s: skin; mu: muscle.

\begin{tabular}{|c|c|c|c|c|c|c|c|}
\hline S. number & Gene & $\mathrm{TPM}_{\mathrm{ma}}$ & $\mathrm{TPM}_{\mathrm{s}}$ & $\mathrm{TPM}_{\mathrm{mu}}$ & $\%$ transcript abundance & $\mathrm{TPM}_{\mathrm{ma}} / \mathrm{TPM}_{\mathrm{s}}$ & $E_{r}$ \\
\hline (1) & $A C B P$ & 64 & 118 & 86 & 0.601 & 0.542 & 0.635 \\
\hline (2) & ACSL1 & 172 & 295 & 363 & 1.616 & 0.583 & 0.526 \\
\hline (3) & ACSS1 & 107 & 29 & 69 & 1.005 & 3.690 & 2.392 \\
\hline (4) & ACSS2 & 226 & 29 & 69 & 2.124 & 7.793 & 5.052 \\
\hline (5) & ADFP $^{*}$ & 474 & 295 & 51 & 4.454 & 1.607 & 3.864 \\
\hline (6) & CD36 & 398 & 29 & 381 & 3.740 & 13.724 & 3.786 \\
\hline (7) & DGAT1 & 32 & 88 & 17 & 0.301 & 0.364 & 0.827 \\
\hline (8) & FABP3 & 755 & 29 & 69 & 7.095 & 26.034 & 16.878 \\
\hline (9) & FASN ${ }^{*}$ & 1499 & 531 & 17 & 14.086 & 2.823 & 15.777 \\
\hline (10) & GPAM $^{*}$ & 312 & 29 & 51 & 2.932 & 10.759 & 8.113 \\
\hline (11) & INSIG1 & 194 & 59 & 17 & 1.823 & 3.288 & 6.126 \\
\hline (12) & LASS2 & 194 & 324 & 17 & 1.823 & 0.599 & 2.614 \\
\hline (13) & $\mathbf{L P L}^{*}$ & 690 & 118 & 138 & 6.484 & 5.847 & 5.407 \\
\hline (14) & $O S B P$ & 21 & 59 & 17 & 0.197 & 0.356 & 0.663 \\
\hline (15) & PLIN & 32 & 29 & 34 & 0.301 & 1.103 & 1.019 \\
\hline (16) & PPARG & 75 & 177 & 17 & 0.705 & 0.424 & 1.367 \\
\hline (17) & SCAP & 21 & 51 & 17 & 0.197 & 0.412 & 0.713 \\
\hline (18) & SCD5 & 1661 & 324 & 51 & 15.608 & 5.127 & 12.921 \\
\hline (19) & SGPL1 & 10 & 177 & 34 & 0.094 & 0.056 & 0.129 \\
\hline (20) & SPTLC1 & 463 & 147 & 34 & 4.351 & 3.150 & 6.549 \\
\hline (21) & SREBF1* & 453 & 88 & 17 & 4.257 & 5.148 & 11.712 \\
\hline (22) & $\mathbf{X D H}^{*}$ & 1186 & 118 & 51 & 11.145 & 10.051 & 15.288 \\
\hline (23) & $A B C A 1$ & 0 & 0 & 0 & 0 & - & - \\
\hline (24) & ABCG2 & 0 & 0 & 0 & 0 & - & - \\
\hline (25) & $A C A C A$ & 10 & 0 & 17 & 0.094 & - & - \\
\hline (26) & AGPAT6 & 593 & 29 & 0 & 5.572 & 20.448 & - \\
\hline (27) & $A S A H L$ & 0 & 0 & 17 & 0 & - & - \\
\hline (28) & $B D H 1$ & 0 & 118 & 51 & 0 & - & 0 \\
\hline (29) & BTN1A1 & 744 & 0 & 0 & 6.991 & - & - \\
\hline (30) & DGAT2 & 0 & 88 & 103 & 0 & - & 0 \\
\hline (31) & FADS1 & 75 & 0 & 51 & 0.705 & - & - \\
\hline (32) & FADS2 & 0 & 0 & 0 & 0 & - & - \\
\hline (33) & INSIG2 & 0 & 0 & 0 & 0 & - & - \\
\hline (34) & LPIN1 & 0 & 0 & 138 & 0 & - & - \\
\hline (35) & OSBPL10 & 140 & 0 & 17 & 1.316 & - & - \\
\hline (36) & OSBPL2 & 0 & 88 & 0 & 0 & - & - \\
\hline (37) & OXCT1 & 10 & 0 & 0 & 0.094 & - & - \\
\hline (38) & PPARGC1A & 21 & 0 & 51 & 0.197 & - & - \\
\hline (39) & PPARGC1B & 0 & 0 & 0 & 0 & - & - \\
\hline (40) & SPHK2 & 0 & 0 & 0 & 0 & - & - \\
\hline (41) & SPTLC2 & 10 & 0 & 17 & 0.094 & - & - \\
\hline (42) & SREBF2 & 0 & 295 & 51 & 0 & - & 0 \\
\hline (43) & THRSP & 0 & 29 & 69 & 0 & - & 0 \\
\hline (44) & UGCG & 0 & 29 & 0 & 0 & - & - \\
\hline$(45)$ & VLDLR & 0 & 0 & 34 & 0 & - & - \\
\hline
\end{tabular}


over skin or muscle; 6 of these 13 genes (ADFP, FASN, $G P A M, L P L, S R E B F 1$, and $X D H)$ further showed preferential expression during adulthood.

The skin has been included for comparison because the mammary tissue is known to be modified cutaneous tissue [9] and differences in the expression pattern of genes between mammary and cutaneous tissue are likely to signify functional differences; muscle tissue has been included as a control. ACSS1, ACSS2, ADFP, CD36, FABP3, FASN, GPAM, INSIG1, LPL, SCD5, SPTLC1, SREBF1, and XDH had higher mammary expression over skin or muscle; ADFP, FASN, GPAM, LPL, SREBF1, and XDH showed preferential expression during adulthood and, hence, was considered most likely to be differentially expressed during milk fat synthesis.

Among genes responsible for fatty acid import into cells, both LPL and CD36 appeared to have greater expression in mammary tissue. $L P L$ primarily functions in the hydrolysis of triglycerides of circulating chylomicrons and very lowdensity lipoproteins (VLDL). CD36 binds long-chain fatty acids and functions in their transport and also as a regulator of fatty acid transport. LPL showed more than 5-fold increase in TPM values in mammary tissue over cutaneous tissue whereas $C D 36$ showed a more than 13 -fold increase. Further, the expression of $L P L$ was greater in adult-derived tissues than in tissues derived from young ones. Our findings support the predication that $L P L$ has higher mammary activity by virtue of high transcript abundance [10]. LPL was the fifth most abundant transcript. Also, more than 8-fold increase in transcript abundance of $C D 36$ has been previously reported during in vivo studies [3].

Among the five genes for acetate/fatty acid activation and intracellular transport, three showed relatively higher expression in mammary tissue. ACSS1 showed a $>3$-fold increase, and ACSS2 showed more than 7-fold increase in transcript abundance. These findings are comparable to previous findings; Bionaz and Loor have reported a higher ( 13-fold) increase in ACSS2 over ACSS1 ( 4-fold) [3]. ACSS1 and ACSS2 are responsible for activation of short-chain fatty acids; while ACSS1, primarily mitochondrial enzyme, activates acetate for energy production, ACSS2, the cytosolic enzyme, activates acetate for fatty acid synthesis [11]. With acetate being the chief substrate for energy production and fatty acid synthesis in the mammary tissue [9], overexpression of ACSS1 and ACSS2 during lactation is teleologically expected. In the same study [3], FABP3 was the second most abundant transcript with a nearly 80 -fold change in transcript abundance at 60 days of lactation. However, the relative change in transcript abundance at onset and 15, 30, 120, and 240 days of lactation ranged about 20-40. In our study, FABP3 showed a $>26$-fold increase and was also the fourth most abundant transcript among all ones considered in the study. $F A B P 3$ is involved in the intracellular trafficking long-chain fatty acids and their acyl coesters.

Fatty acid synthesis and desaturation per se are the most important step in milk fat synthesis. However, of the five genes studied, only two appear to be involved during the milk fat synthesis response in the mammary tissue. FASN that catalyzes the formation of long-chain fatty acids from acetyl-CoA, malonyl-CoA, and NADPH was the second most abundant transcript and showed $\sim 3$-fold increase in expression. SCD5, responsible for introducing a double bond in fatty acyl-coenzyme $\mathrm{A}$ at the delta 9 position, was the most abundant transcript $(\sim 15.6 \%)$ with more than 5 -fold increase in mRNA expression. Bionaz and Loor have also reported SCD5 to be the most abundant ( 23\%) among transcripts of genes involved in milk fat synthesis. However, in their study, the relative increase in expression has been reported to be much higher ( 10-40-fold increase) [3].

GPAM, with more than $2 \%$ of all transcripts studied, was the only one of five genes involved in triacylglycerol synthesis found to be overexpressed ( $>10$-fold increase). Bionaz and Loor have reported identical values of transcript abundance and relative expression of this gene [3]. Among the genes involved in sphingolipid synthesis, SPTLC1 appeared to be overexpressed (>3-fold) whereas the expression of SGPL1 appeared to be downregulated at about 1/20th of cutaneous expression.

Among the genes involved in lipid droplet formation, $A D F P$ and $X D H$ were overexpressed with 1.6- and a 10fold increase in relative expression, respectively, over the cutaneous tissue. Both of these genes also showed preferential expression in adult-derived tissues. $X D H$ includes xanthine dehydrogenase and xanthine oxidase; the enzyme can be converted from the dehydrogenase form (D) into the oxidase form (O) irreversibly by proteolysis or reversibly through the oxidation of sulfhydryl groups. $X D H$ was the third most abundant of all transcripts $(>11 \%)$. Bionaz and Loor have similarly reported $>7 \%$ abundance of $X D H$ transcripts and about 8 -fold increase in its relative expression in the lactating mammary tissue [3].

Among transcriptional regulators that drive or sustain milk fat synthesis, INSIG1 and SREBF1 appeared to be overexpressed. Percent transcript abundance and relative increase in expression for the genes were about $1.8 \%, \sim 3$-fold, and $4.2 \%, \sim 5$-fold, respectively; 2.4 - and 2.5-fold increases in the expression of these two genes have been reported previously [12]. Increase in INSIG1 [3] and SREBF1 [13] activities during lactation to much greater extents than being reported in the present study have also been reported earlier. A greater function of SREBF2 than SREBF1 in milk fat synthesis has been hypothesized [3]. Our study could not include SREBF2 due to insufficient information on this gene in the UniGene ESTProfile. However, based on our results, SREBF1 is expected to play a role at least equivalent to, if not greater than, $S R E B F 2$ in regulating the transcriptional response during milk fat production in the mammary tissue. None of the genes involved in xenobiotic and cholesterol transport and ketone body utilization appeared to be differentially expressed as part of the lactational milk fat synthesis response.

3.3. Gene Interaction Networks. Interaction network for all the 45 genes, obtained using STRING, has been shown in Figure 2. The interactions were further purged to map only those 13 genes that showed preferential expression in mammary tissue in UniGene ESTProfile (Figure 3); in Figure 3(a), the weight of the edges shows the strength of 


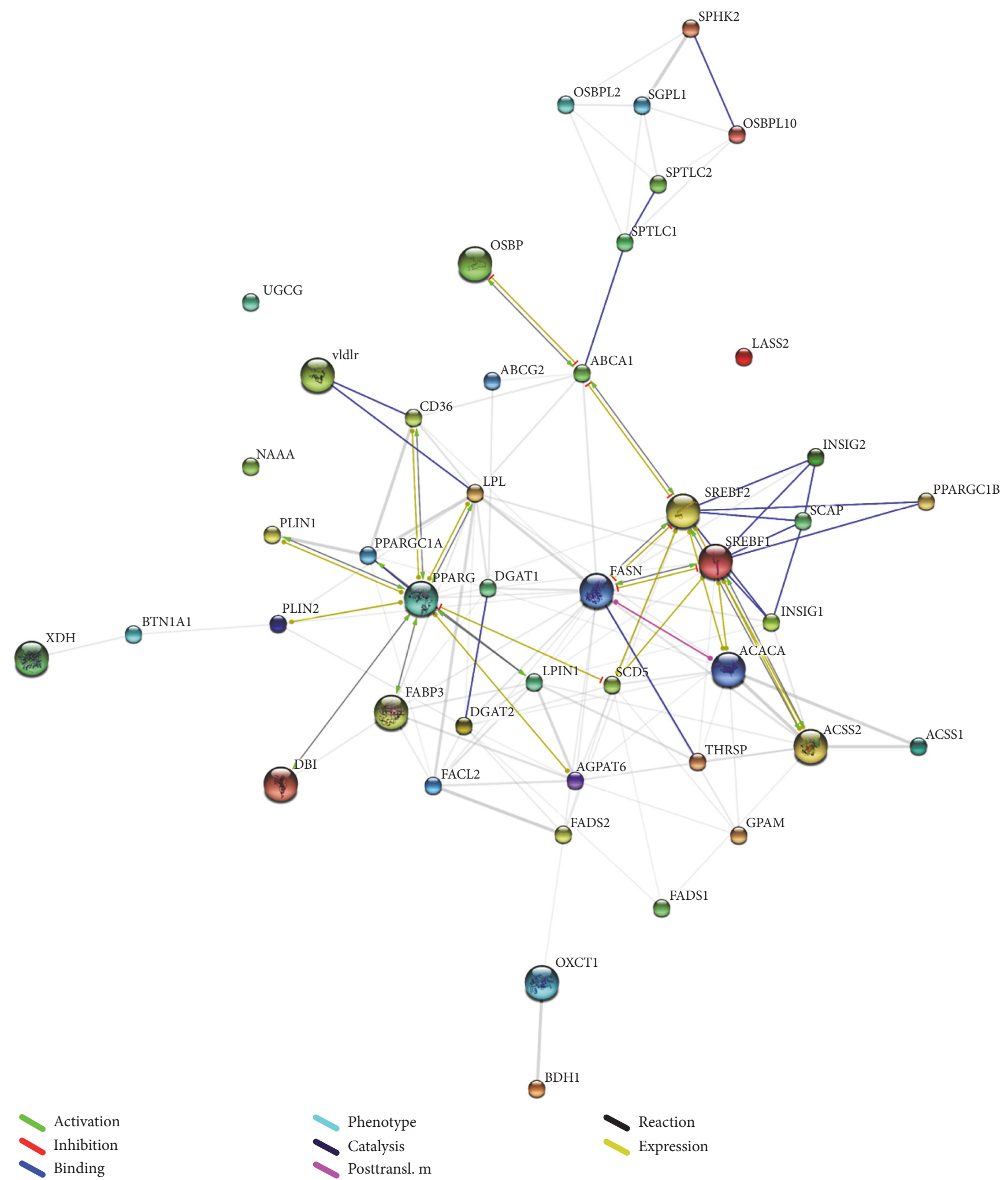

FIGURE 2: Interaction network of the genes involved in milk fat biosynthesis and secretion. STRING $v 9.1$ was used to derive the network among genes involved in milk fat synthesis and secretion. FASN appears to be the central component in milk fat synthesis. The entire network appears to operate under two different control systems: one under PPARG and another under the joint control of SREBF1 and SREBF2. Three genes ( $A S A H L / N A A A, L A S S 2$, and UGCG) involved in sphingolipid synthesis did not interact with any other gene/gene product in the network. 


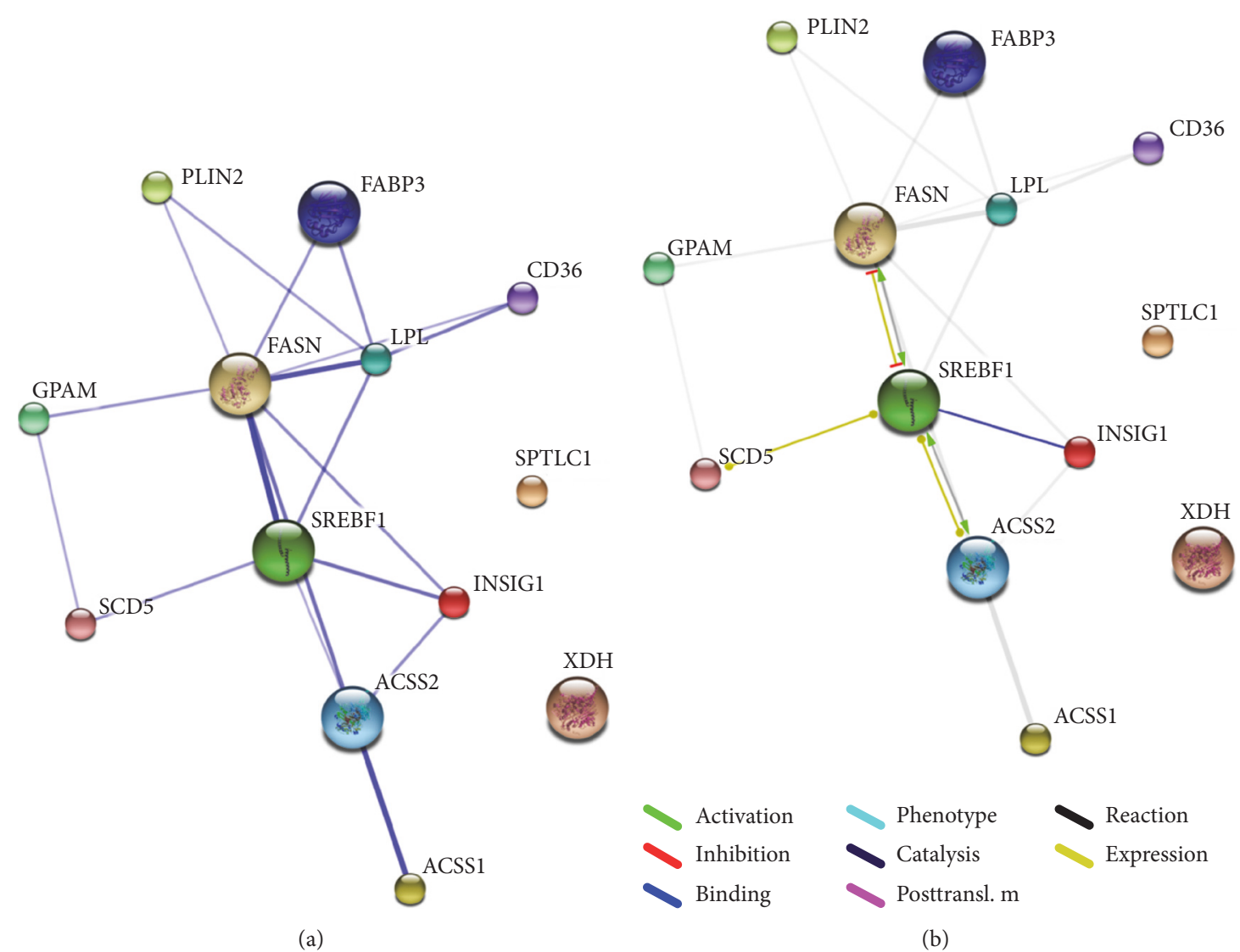

FIGURE 3: Interaction network of overexpressed genes. STRING $v 9.1$ was also used to map the interaction networks between the genes that appeared to be overexpressed based on transcript abundance studies. In (a), the weight of the edges represents the confidence of the interaction; the nature of these interactions has been shown in (b). SPTLC1 and XDH did not interact with any other gene of the 11 genes.

the interactions. The nature of these interactions has been depicted in Figure 3(b).

Network analysis shows that FASN, SREBF1, SREBF2, $P P A R G$, and ACSS2 are the major components of the milk fat synthesis pathway. Two subnetworks are evident: one under the predominant control of $P P A R G$ and the other one majorly under the joint control of SREBF1 and SREBF2; both these subnetworks appear to converge at FASN. SCD5, the most abundant transcript, was the only gene under the direct control of PPARG, SREBF1, and SREBF2. Also, three of the four genes showing the maximum relative change in expression, namely, $F A B P 3, C D 36$, and $X D H$, were chiefly under the control of PPARG. Thus, PPARG, though not found to be overexpressed based on TPM values, appears to play a major role in the transcriptional regulation of milk fat synthesis. Bionaz and Loor [3] have also advocated a role of PPARG in regulating the entire bovine milk fat synthesis machinery notwithstanding its downregulation and low mRNA abundance in mammary tissue. The genes involved in sphingolipid synthesis and ketone body utilization appeared to form two nearly independent clusters with sparse interaction with the rest of the network; ASAHL (NAAA), LASS2, and UGCG did not interact with any other gene at all. THRSP did not form part of the cluster of genes involved in transcriptional regulation. While all other gene products were involved directly or indirectly in interactions with each other, SPTLC1 and $X D H$ did not interact with any of these gene products.

STRING was also used to determine coexpression patterns between these genes; a functional association of the gene products can be assumed if a group of genes exhibits strong coexpression. Only a low level of association could be inferred between some of the genes based on the coexpression pattern (Figure 4). Again, FASN appeared to be the central component of the milk fat synthesis pathway.

To conclude, in this study we have put forward a simplistic approach for determining the relative expression of genes based on their transcript abundance values in UniGene ESTProfile. Further, we used this approach for the expression profiling of genes involved in milk fat biosynthesis and secretion in bovines. Based on our findings, an updated model of the transcriptional profile of the genes involved in milk fat production by the mammary gland has been presented. For the genes studied, the results were in good agreement with the previously reported results from wetlab studies, indicating the satisfactory performance of our computational approach. Our study included cutaneous tissue as a control assuming its ontogenetic equivalence to the quiescent, nonlactating mammary gland; the congruity of our findings 


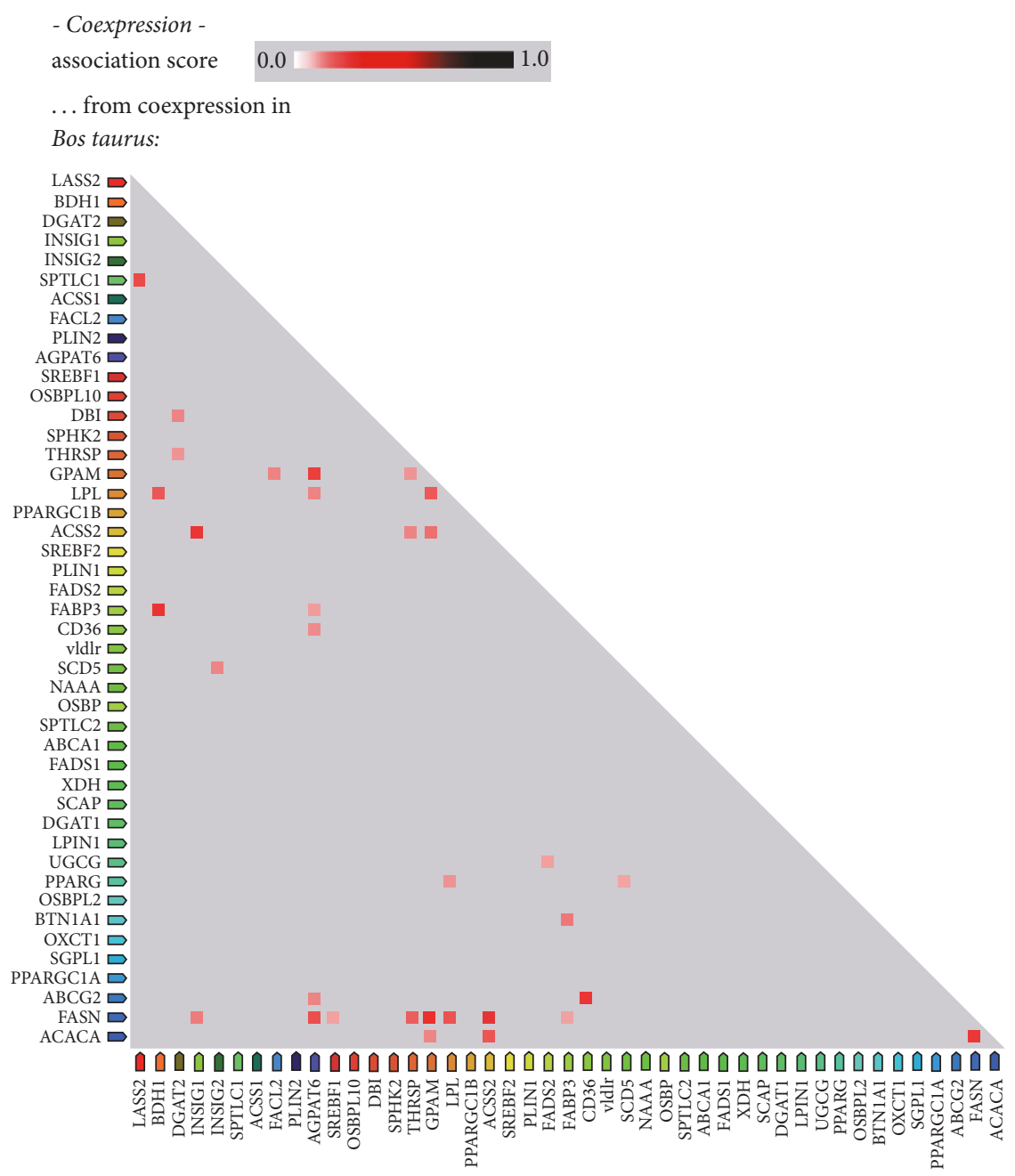

(a)

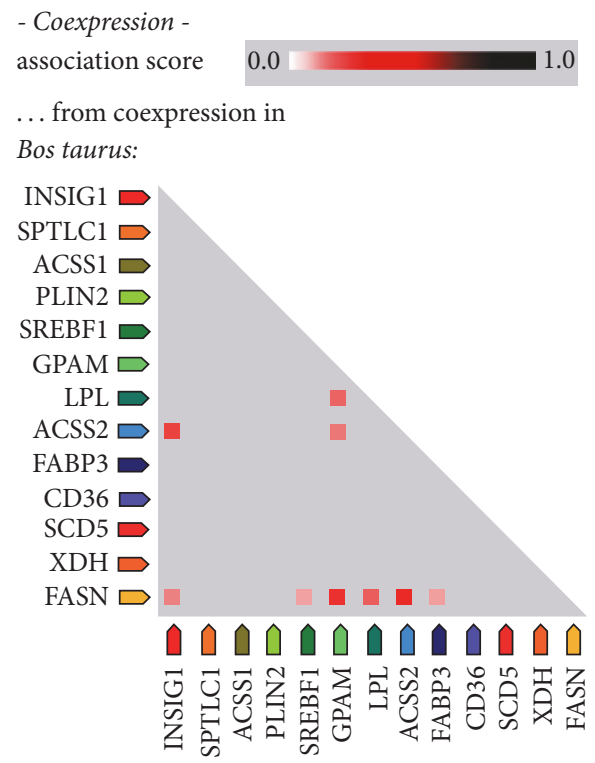

(b)

FIGURE 4: Coexpression of genes involved in milk fat biosynthesis and secretion. Coexpression pattern of the genes involved in milk fat synthesis and secretion in bovines (a) was derived from STRING. Analysis of the coexpression pattern of the thirteen genes that appeared to be overexpressed based on transcript abundance (b) showed a weak association between these genes. FASN shows coexpression based functional association with the maximum number of genes in both (a) and (b).

with those from previous studies projects this equivalence beyond the histological landscape to a biomolecular level. Previously, SREBF2 has been upheld as the major regulator of transcription during milk fat biosynthesis, refuting the role of SREBF1. Our results reinstate SREBF1 as a major transcriptional regulator, along with INSIG1, during the process. Using interaction network analysis of the genes, we could also show two separate transcriptional controls under $P P A R G$ and SREBFs. FASN, SREBF1, SREBF2, PPARG, and ACSS2 were the major components of the milk fat synthesis pathway. However, expression profiles could not be studied for nearly half of the genes due to incomplete UniGene ESTProfile. Also, the inferences would have been more conclusive if UniGene ESTProfile also included information on the stage of lactation during which the mammary glands had been sampled. Thus, further studies are warranted to verify the proposed model and to fill in the research gaps in the present study.

\section{Conflicts of Interest}

The authors declare no conflicts of interest.

\section{Acknowledgments}

Bhaskar Ganguly acknowledges the financial assistance received from Council of Scientific and Industrial Research (CSIR), India, in the form of a Senior Research Fellowship during this study.

\section{References}

[1] A. Tăbăran, V. A. Balteanu, E. Gal et al., "Influence of DGAT1 K232A Polymorphism on Milk Fat Percentage and Fatty Acid Profiles in Romanian Holstein Cattle," Animal Biotechnology, vol. 26, no. 2, pp. 105-111, 2015. 
[2] D. Murray, P. Doran, P. MacMathuna, and A. C. Moss, "In silico gene expression analysis - An overview," Molecular Cancer, vol. 6, article no. 50, 2007.

[3] M. Bionaz and J. J. Loor, "Gene networks driving bovine milk fat synthesis during the lactation cycle," BMC Genomics, vol. 9, no. 1, article 366, 2008.

[4] J. Stalker, B. Gibbins, P. Meidl et al., "The Ensembl web site: Mechanics of a genome browser," Genome Research, vol. 14, no. 5, pp. 951-955, 2004.

[5] M. S. Boguski, T. M. J. Lowe, and C. M. Tolstoshev, "dbEST database for "expressed sequence tags"," Nature Genetics, vol. 4, no. 4, pp. 332-333, 1993.

[6] Z. Zhang, S. Schwartz, L. Wagner, and W. Miller, "A greedy algorithm for aligning DNA sequences," Journal of Computational Biology, vol. 7, no. 1-2, pp. 203-214, 2000.

[7] U. J. Pontius, L. Wagner, and G. D. Schuler, "UniGene: A Unified View of the Transcriptome," in The NCBI Handbook. Bethesda, MD: National Library of Medicine (US), NCBI, 2003.

[8] A. Franceschini, D. Szklarczyk, S. Frankild et al., "STRING v9.1: protein-protein interaction networks, with increased coverage and integration," Nucleic Acids Research, vol. 41, no. 1, pp. D808D815, 2013.

[9] S. C. Park and G. L. Lindberg, "The mammary gland and lactation," in Duke's Physiology of Domestic Animals, W. O. Reece, Ed., pp. 720-741, Panima Publishing Corp, New Delhi, 12th edition, 2005.

[10] L. Bernard, C. Richard, V. Gelin, C. Leroux, and Y. Heyman, "Milk fatty acid composition and mammary lipogenic genes expression in bovine cloned and control cattle," Livestock Science, vol. 176, pp. 188-195, 2015.

[11] T. Fujino, J. Kondo, M. Ishikawa, K. Morikawa, and T. T. Yamamoto, "Acetyl-CoA Synthetase 2, a Mitochondrial Matrix Enzyme Involved in the Oxidation of Acetate," The Journal of Biological Chemistry, vol. 276, no. 14, pp. 11420-11426, 2001.

[12] Y. Gao, X. Lin, K. Shi, Z. Yan, and Z. Wang, "Bovine mammary gene expression profiling during the onset of lactation," PLoS ONE, vol. 8, no. 8, Article ID e70393, 2013.

[13] K. J. Harvatine and D. E. Bauman, "SREBP1 and thyroid hormone responsive spot 14 (S14) are involved in the regulation of bovine mammary lipid synthesis during diet-induced milk fat depression and treatment with CLA," Journal of Nutrition, vol. 136, no. 10, pp. 2468-2474, 2006. 

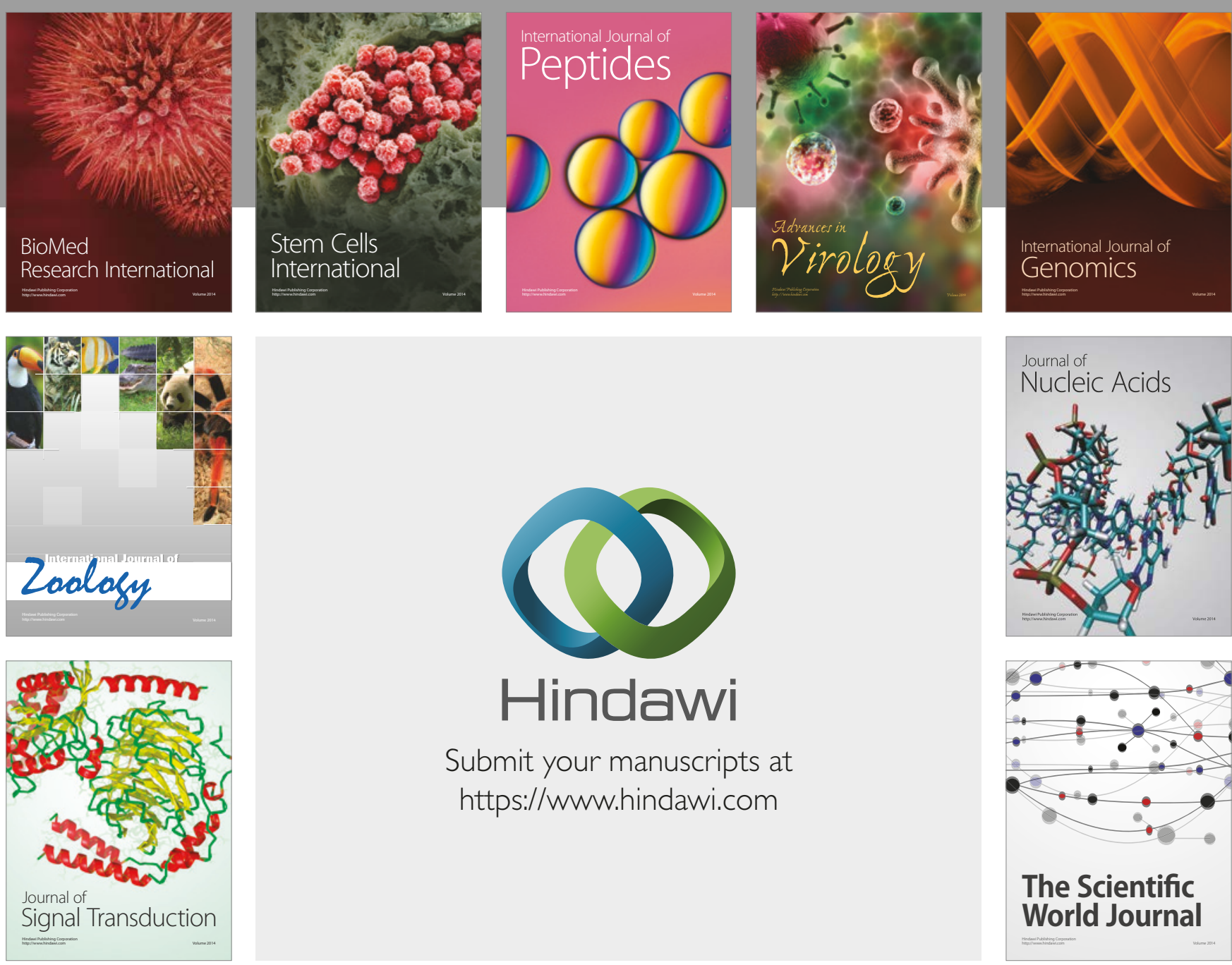

Submit your manuscripts at

https://www.hindawi.com
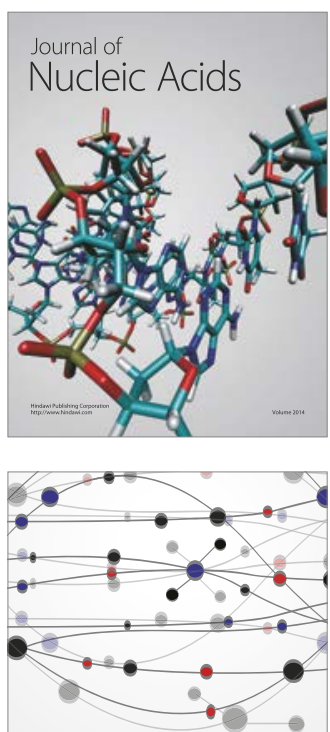

The Scientific World Journal

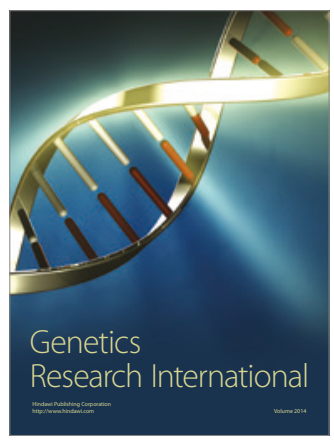

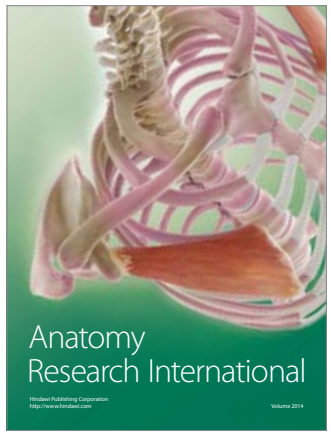

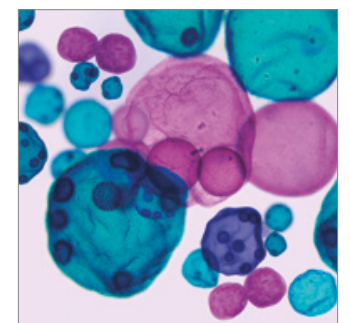

International Journal of Microbiology
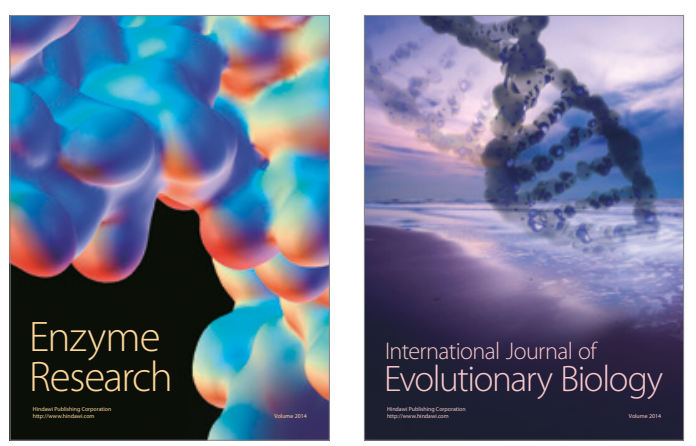
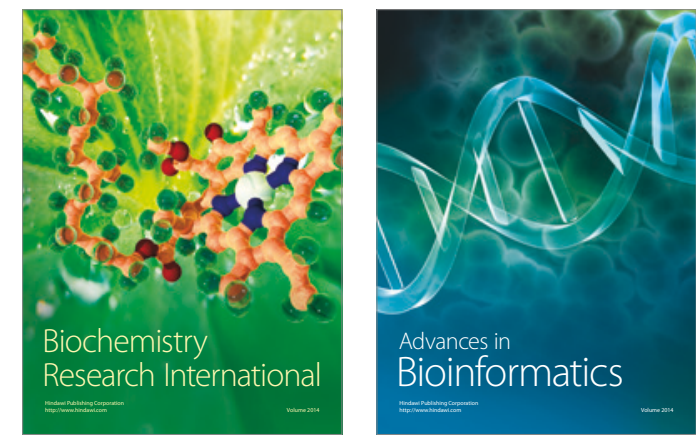

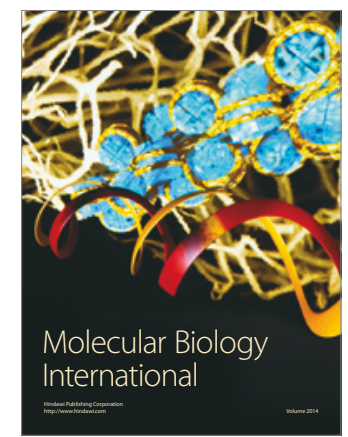

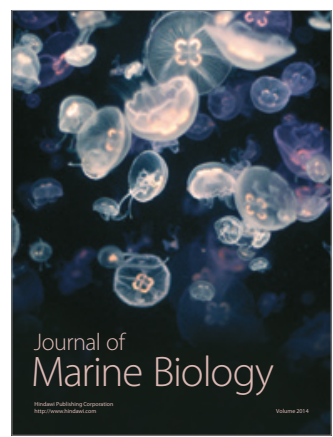

